Research Article

\title{
Oxidative Stress Risk Is Increased with a Sedentary Lifestyle during Aging in Mexican Women
}

\author{
Martha A. Sánchez-Rodríguez $\left(\mathbb{D},{ }^{1}\right.$ Mariano Zacarías-Flores $\mathbb{D}^{2},{ }^{2}$ Elsa Correa-Muñoz $\left(\mathbb{D},{ }^{1}\right.$ \\ Alicia Arronte-Rosales, ${ }^{1}$ and Víctor Manuel Mendoza-Núñez iD ${ }^{1}$ \\ ${ }^{1}$ Research Unit on Gerontology, FES Zaragoza, National Autonomous University of Mexico, Mexico City, Mexico, Av. Guelatao \\ No. 66, Col. Ejército de Oriente, Iztapalapa, Ciudad de Mexico, CP 09230, Mexico \\ ${ }^{2}$ Division of Obstetrics and Gynecology, Hospital Gustavo Baz Prada, Institute of Health of the State of Mexico, Nezahualcóyotl, \\ State of Mexico CP 57300, Mexico
}

Correspondence should be addressed to Víctor Manuel Mendoza-Núñez; mendovic@unam.mx

Received 1 April 2021; Revised 1 September 2021; Accepted 5 October 2021; Published 25 October 2021

Academic Editor: Lillian Barros

Copyright (c) 2021 Martha A. Sánchez-Rodríguez et al. This is an open access article distributed under the Creative Commons Attribution License, which permits unrestricted use, distribution, and reproduction in any medium, provided the original work is properly cited.

\begin{abstract}
Oxidative stress (OS) increases during the human aging process, and the sedentary lifestyle could be a prooxidant factor. In this study, we determine the effect of sedentary lifestyle on OS during the aging process in Mexican women. A longitudinal study of two-year follow-up was carried out with 177 community-dwelling women (40-69y) from Mexico City. We measured as OS markers plasma malondialdehyde, erythrocyte glutathione peroxidase (GPx) and superoxide dismutase (SOD), total plasma antioxidant status, uric acid level, antioxidant gap, and SOD/GPx ratio. To define OS using all the markers, we defined cut-off values of each parameter based on the $90^{\text {th }}$ percentile of young healthy subjects and, we calculated a stress score (SS) ranging from 0 to 7 , which represented the intensity of the marker modifications. All the women answered a structured questionnaire about prooxidant factors, including physical activity specially the type of activity, frequency, and duration, and they answered Spanish versions of self-assessment tests for establishing dysthymia and insomnia as potential confounders. Principal component and Poisson regression analysis were used as statistical tools, being two-year OS the primary outcome. The OS was considerate as $\mathrm{SS} \geq 4$ and sedentary lifestyle as $<30 \mathrm{~min}$ /day of physical activity, beside several prooxidant factors and age that were covariables. SS is higher in sedentary lifestyle women after the two-year follow-up; although, the difference was statistically significant only in older women. Four principal components were associated with the OS, and 7 out of 8 prooxidant factors were important for the analysis, which were included in the Poisson model. The predictive factors for OS were the sedentary lifestyle (adjusted $\mathrm{PR}=2.37, \mathrm{CI}_{95 \%}: 1.30-4.30, p<0.01$ ), and age, in which the risk increases 1.06 $\left(\mathrm{CI}_{95 \%}: 1.02-2.11, p<0.01\right)$ by each year of age. Our findings suggest that a sedentary lifestyle increases the OS during the aging in Mexican women.
\end{abstract}

\section{Introduction}

Since 1956, it was proposed that the aging is a biological process of every living organism which is related to oxidative stress (OS) [1], and this is defined as a biochemical imbalance between oxidants and antioxidant systems that produces an oxidative damage in biomolecules [2]. In humans, many investigations have been carried out to demonstrate an OS increment during the aging process [3, 4]; besides, there have been indicated several factors that accelerate or mitigate the relationship. Of these last factors, the intake of natural and artificial antioxidants, furthermore, the healthy lifestyle has been described as actions that promote healthy aging with less OS [5].

In women, the main changes produced by biological aging are associated with ovarian dysfunction during the menopausal transition with low production of estrogens, which causes many discomforting signs and symptoms such as hot flashes, night sweats, depression, headaches, insomnia, and lack of energy, among others $[6,7]$. Most of these 
are prooxidant factors; thus, they favor the oxidation of biomolecules increasing the OS [8-10]. Likewise, as menopausal transition takes place, the women experience changes in their physical functioning which leads them to adopt to a more sedentary lifestyle [11]. Today, sedentary behavior is a characteristic of the population in general. This behavior has been recognized as a risk factor for a variety of diseases, mainly cardiovascular disease, and as a prooxidant risk factor [12].

Although this behavior is more prevalent in men than in women, the women acquire a sedentary lifestyle as the age progresses, starting around the fifth decade of life and is associated with the menopause $[13,14]$, as we indicated above. As other lifestyle factors, the sedentary lifestyle has been pointed out as a prooxidant factor because it has been related to the dysregulation of cellular redox status and diminished mitochondrial function [15]. Likewise, a study with muscle biopsies of sedentary aged humans has shown high intramuscular lipid peroxidation at rest and during acute exercise, finding the association between sedentary behavior, aging, and OS [16]. Although few studies confirm this relationship at the population level and much less in female aging, because the studies have focused mainly on men of different age groups or in older subjects, besides, these researchers have studied the antioxidant effect of physical activity. Thus, the aim of this study was to determine the effect of sedentary lifestyle on OS during the aging process in Mexican women.

\section{Materials and Methods}

2.1. Study Design and Participants. An observational longitudinal study was carried out with 177 women (40-69y) from Mexico City (healthy or with medical conditions under control namely hypertension and/or diabetes). The women were invited to participate in the Menopause and Oxidative Stress Project from the Research Unit on Gerontology at National Autonomous University of Mexico, Zaragoza Campus, from March to July 2017. The invitation was made through brochures specifying the eligibility criteria and objectives of the study. For this call, 558 women attended, and 383 potential participants were eliminated for different reasons such as the women with cardiovascular disease, hormonal or antioxidant therapy, or out of the age group, among others (Figure 1). The women with cardiovascular, kidney, hepatic, and cancer disease, assessed by medical history and physical examination, were eliminated. The women were separated into three groups according to decades of age: (i) 59 young women $(40-49 y)$, (ii) 76 midlife women (50-59y), and (iii) 42 older women (60-69y). The participants had not previously taken any antioxidant supplement or hormone therapy, for at least six months prior to the beginning and throughout the study. The follow-up was for two years.

All participants agreed and signed an informed consent in order to participate in the study. This work is part of a clinical trial approved by the Ethics Committee of the National Autonomous University of Mexico (UNAM) Zaragoza Campus (register number FESZ/DEPI/CI/004/17) and recorded in the ISRCTN Registry Survey (ISRCTN14939779). In this study, follow-up of participants in the no intervention arm is shown.

To establish their health status, the participants were subjected to the following examinations: complete blood count, lipid profile and glucose, blood pressure, and anthropometric measurements, in addition to a complete clinical history. We used the cut-off points of reference values for Mexican population [17].

After 12-h fasting period, blood samples were collected by venipuncture. The samples were placed in vacutainer/siliconized test tubes with heparin as anticoagulant agent and a separating gel with no additives (Becton-Dickinson, Mexico City, Mexico). With the heparinized samples, we carried out the complete blood count in a Celly 70 auto analyzer (Chronolab, Mexico City, Mexico). The serum chemical measurements of glucose, total cholesterol, triglycerides, and high-density lipoprotein cholesterol (HDL-c) levels were tested using a Cobas C111 analyzer (Roche Diagnostics, Basilea, SW). As quality control, the intra- and interassay variation coefficients were obtained, which were less than $5 \%$ in all determinations.

The weight of each woman was measured in a fasting state (after evacuation), and they wore a clinic gown and underwear using a Torino ${ }^{\circledR}$ scale (Tecno Lógica, Mexicana, Mexico, TLM $^{\circledR}$ ) calibrated before each weight measurement. The height was obtained with an aluminum cursor stadiometer (graduated in millimeters). For this, the woman was placed on the stadiometer in the Frankfurt horizontal plane, and she stood barefoot, backward, and with the head in contact with it. We calculate body mass index (BMI) by dividing weight (in kilograms) through squared height (in meters). In accordance with international guidelines, the BMI cut-off value was equal or greater than $25.0 \mathrm{~kg} / \mathrm{m}^{2}$ [18]. A mercurial manometer was used to measure each woman blood pressure in both arms in the morning, in sitting position after resting for 5 minutes and in a fasting condition; the mean of these measurements was reported as result. All the measurements were carried out by medical technicians trained in previous sessions for standardization of the procedures (intertechnician kappa coefficient $=0.95$, $p<0.0001$ ), and they were monitored to avoid biases in the measurements.

2.2. Sample Size. The sample size was calculated based on a one mean known principle detect a difference of 0.25 stress score (SS) points like the results found in a 6-month interventional study of low impact exercise vs. nonintervention [19]. To compute the sample size with the tables for clinical studies [20], a power of $80 \%$ and a $5 \%$ level of significance were used because it is a longitudinal study, and it was added $20 \%$ more to compensate possible lost during the follow-up. The final size was 154 women, but we selected 177 women who meet the eligibility criteria to ensure the results.

2.3. Oxidative Stress Measurement. With the heparinized samples, we measured plasma malondialdehyde level (MDA), red blood cell glutathione peroxidase (GPx), superoxide dismutase (SOD) activities, and plasma total antioxidant 


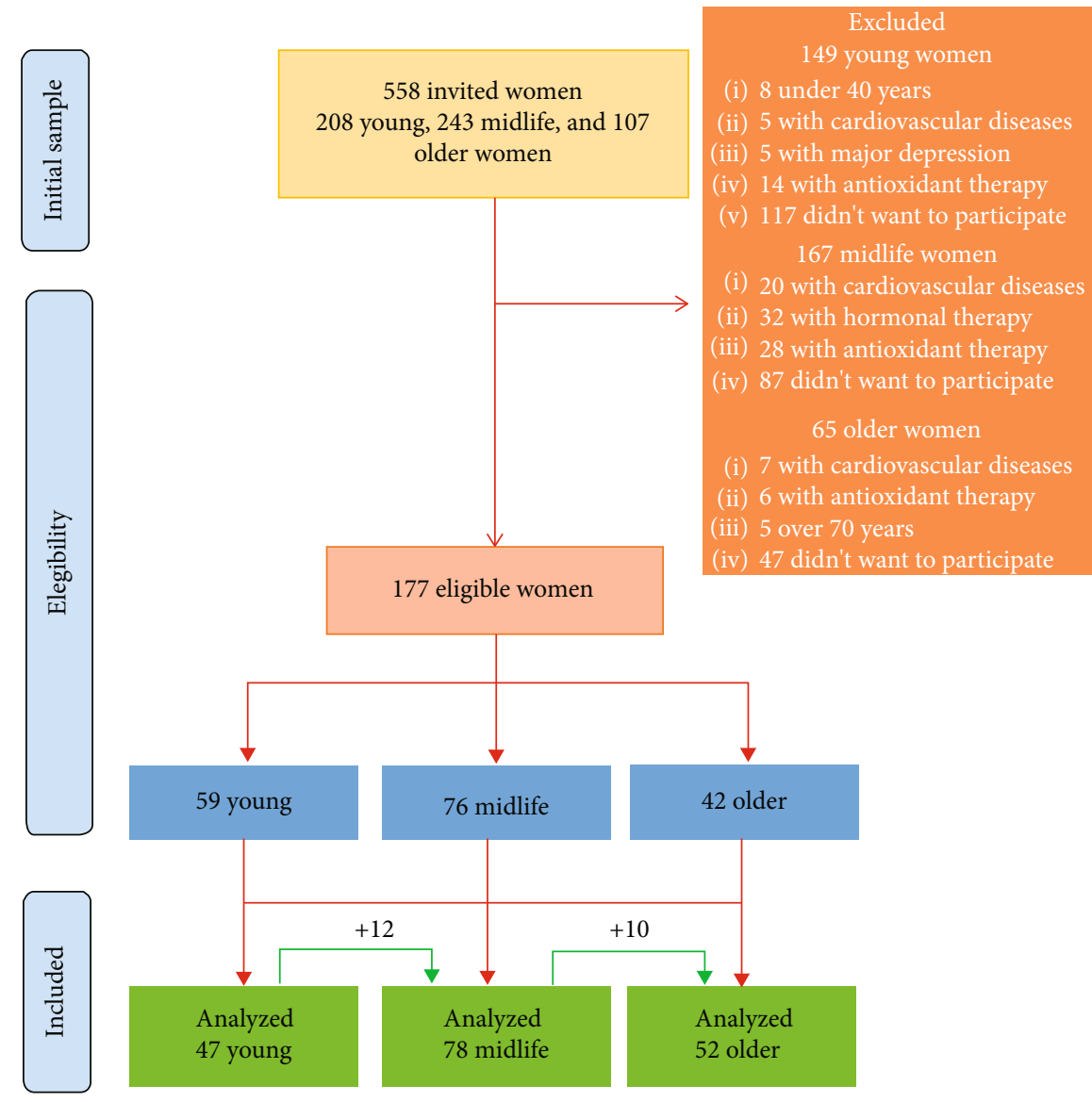

Figure 1: Flow diagram of the study.

status (TAS). In our research laboratory, the methods were validated (within run precision values: $6 \%, 4.6 \%, 3.8 \%$, and $4.3 \%$, respectively). We added $10 \mu \mathrm{L}$ of $2 \mathrm{mM}$ butylated hydroxytoluene in ethanol at $95 \%$ immediately after centrifugation, and artificial formation of thiobarbituric acid reacting substances (TBARS) in the samples was prevented.

The MDA level was measured using the TBARS assay described by Jentzsch et al. [21]. This procedure was previously validated by our research group. To avoid the amplification of peroxidation during the assay, the chain-breaking antioxidant butyryl hydroxytoluene (BHT) (Sigma Chemical Co. St. Louis, MO, USA) was added to the heparinized sample. Briefly, in this procedure, $400 \mu \mathrm{L}$ of sample or $1,1,3,3$ tetramethoxypropane (TMP) (Sigma Chemical Co.), used as MDA standard $(0.2-4 \mathrm{mmol} / \mathrm{L})$, was mixed with $400 \mu \mathrm{L}$ orthophosphoric acid ( $0.2 \mathrm{~mol} / \mathrm{L}$, Sigma Chemical Co.) and $50 \mu \mathrm{L}$ BHT $(2 \mathrm{mmol} / \mathrm{L})$. After $50 \mu \mathrm{L}$ of thiobarbituric acid (TBA) $(0.11 \mathrm{~mol} / \mathrm{L}$ in $0.1 \mathrm{~mol} / \mathrm{L} \mathrm{NaOH}$; Fluka Chem., Buchs, Switzerland) reagent was added, the mix was placed in a water bath at $90^{\circ} \mathrm{C}$ for $45 \mathrm{~min}$. After cooled in ice to stop the reaction, TBARS were extracted with $1000 \mu \mathrm{L} n$-butanol (Sigma Chemical Co.). The butanol phase was read at $535 \mathrm{~nm}$ and $572 \mathrm{~nm}$ to correct baseline absorption. The absorption difference was used to calculate MDA concentration in the samples using the calibration curve.

GPx, SOD activities, and total antioxidant status (TAS) were performed using commercial kits according to the manufacturer's instructions (Randox Laboratories, Ltd.). In GPx test, cumene hydroperoxide in the presence of glutathione reductase and NADPH produces the oxidation of glutathione, which is immediately converted into the reduced form with the subsequent oxidation of NADPH to NADP+, which is measured at $340 \mathrm{~nm}$. In the method to determinate SOD activity, superoxide radicals were generated using xanthine and xanthine oxidase; the superoxide radicals react with 2-(4-iodophenyl)-3-(4-nitrophenol)-5-phenyltetrazolium chloride to form a red formazan dye. The TAS test determines the decrease in absorbance that is proportional to the blood antioxidant concentration of the sample, and the method measures 2,2-azino-bis (3-ethylbenzthiazoline6-sulfonic acid, $\mathrm{ABTS}^{+}$) radical formation kinetics. All the measurements were performed in a Shimadzu UV-1601 UV-Vis spectrophotometer (Kyoto, Japan). Also, uric acid by the uricase colorimetric method and albumin levels using bromocresol green technique, with a Cobas C111 analyzer, were assessed. The intrarun precision assays were $2.8 \%$ and $3.1 \%$, respectively.

Additionally, we obtained the SOD/GPx ratio and the antioxidant gap (GAP) with the follow equation:

GAP $=$ TAS $-[($ albumin $(\mu \mathrm{mol}) \times 0.69)+$ uric acid $(\mu \mathrm{mol})][22]$.

To define OS using all the markers, we defined cut-off values of each parameter based on the $90^{\text {th }}$ percentile of young healthy subjects. The cut-off values were 
TABLE 1: Baseline characteristics of study groups.

\begin{tabular}{|c|c|c|c|}
\hline Parameter & Young women $(n=59)$ & Midlife women $(n=76)$ & Older women $(n=42)$ \\
\hline Age (years) & $46 \pm 2$ & $54 \pm 3^{\mathrm{a}}$ & $64 \pm 3^{\mathrm{b}}$ \\
\hline Hemoglobin $(\mathrm{g} / \mathrm{dL})$ & $14.1 \pm 1.4$ & $14.4 \pm 1.1$ & $14.6 \pm 0.9$ \\
\hline Glucose $(\mu \mathrm{mol} / \mathrm{L})$ & $5.3 \pm 2.0$ & $5.9 \pm 2.5$ & $6.7 \pm 3.2^{\mathrm{c}}$ \\
\hline Cholesterol $(\mu \mathrm{mol} / \mathrm{L})$ & $5.6 \pm 1.0$ & $5.6 \pm 1.2$ & $5.8 \pm 1.3$ \\
\hline Triglycerides $(\mu \mathrm{mol} / \mathrm{L})$ & $2.1 \pm 1.1$ & $2.1 \pm 1.4$ & $2.5 \pm 2.4$ \\
\hline $\operatorname{HDLc}(\mu \mathrm{mol} / \mathrm{L})$ & $1.5 \pm 0.4$ & $1.4 \pm 0.4$ & $1.4 \pm 0.4$ \\
\hline Body mass index $\left(\mathrm{kg} / \mathrm{m}^{2}\right)$ & $28.82 \pm 4.4$ & $28.88 \pm 5.0$ & $27.89 \pm 4.8$ \\
\hline Systolic blood pressure ( $\mathrm{mm} \mathrm{hg)}$ & $117 \pm 13$ & $123 \pm 15$ & $135 \pm 28^{\mathrm{b}, \mathrm{d}}$ \\
\hline Diastolic blood pressure ( $\mathrm{mm} \mathrm{hg}$ ) & $79 \pm 8$ & $81 \pm 8$ & $82 \pm 14$ \\
\hline Smoking ( $\geq 2$ cigarettes/d) & $8(14 \%, 6-22 \%)$ & $7(9 \%, 3-15 \%)$ & $5(12 \%, 2-22 \%)$ \\
\hline Highly caffeine beverage intake ( $\geq 2$ cups/d) & $20(34 \%, 22-46 \%)$ & $31(41 \%, 30-52 \%)$ & $22(52 \%, 37-67 \%)$ \\
\hline Alcohol beverages intake ( $\geq 2$ cups/d) & $6(10 \%, 2-18 \%)$ & $8(11 \%, 3-18 \%)$ & $6(15 \%, 4-26 \%)$ \\
\hline Sedentary lifestyle $(<30 \mathrm{~min}$ of physical activity/d) & $27(46 \%, 33-59 \%)$ & $36(47 \%, 36-58 \%)$ & $14(33 \%, 19-47 \%)$ \\
\hline Insomnia (Athens insomnia scale $>8$ ) & $23(39 \%, 27-51 \%)$ & $44(56 \%, 45-67 \%)$ & $16(38 \%, 23-53 \%)$ \\
\hline Dysthymia & $14(24 \%, 13-35 \%)$ & $24(32 \%, 22-42 \%)$ & $13(31 \%, 17-45 \%)$ \\
\hline Diabetes mellitus & $4(7 \%, 1-14 \%)$ & $11(15 \%, 7-23 \%)$ & $12(29 \%, 15-43 \%)^{\mathrm{e}}$ \\
\hline Arterial hypertension & $16(27 \%, 16-38 \%)$ & $32(42 \%, 31-53 \%)$ & $26(62 \%, 47-77 \%)^{\mathrm{e}, \mathrm{f}}$ \\
\hline
\end{tabular}

Continuous data show means \pm standard deviation; categorical data show frequency, percentage. and $95 \%$ confidence interval. One-way ANOVA test with Tukey test as posthoc. ${ }^{\mathrm{a} Y o u n g}$ vs. midlife, $p<0.0001$; ${ }^{\mathrm{b}}$ young vs. older, $p<0.0001$; ${ }^{\mathrm{c}}$ young vs. older, $p<0.05$; ${ }^{\mathrm{d}}$ midlife vs. older, $p<0.01$. $\chi^{2}$ test, ${ }^{\mathrm{e}}$ young vs. older, $p<0.0001$; ${ }^{\mathrm{f}}$ midlife vs. older, $p<0.05$.

$\mathrm{MDA} \geq 0.320 \mu \mathrm{mol} / \mathrm{L}, \mathrm{GPx} \leq 50.1 \mathrm{U} / \mathrm{gHb}, \mathrm{SOD} \leq 1.20 \mathrm{U} / \mathrm{gHb}$, $\mathrm{TAS} \leq 1030 \mu \mathrm{mol} / \mathrm{L}, \mathrm{SOD} / \mathrm{GPx} \geq 0.023$, and GAP $\leq 278 \mu \mathrm{mol} / \mathrm{L}$. The cut-off values of the uric acid were the median of the reference interval $(>268 \mu \mathrm{mol} / \mathrm{L}$ for adult woman and $315 \mu \mathrm{mol} / \mathrm{L}$ for an older woman) as determined to the Mexican population [17]. A score of 1 was given to each value higher or lower than the cut-off point established, and then we sum all the parameters, computing an oxidative stress score (SS) ranging from 0 to 7 , which represented the intensity of the marker modifications. A cut-off value of $\geq 4$ was considered as OS, which is the median value of SS as previously was proposed [23].

2.4. Evaluation of Dysthymia, Insomnia, and Prooxidant Lifestyle Factors. The women answered Spanish versions of self-assessment tests for established the possibility of dysthymia and insomnia as potential confounders and a structured questionnaire about prooxidant factors to know about their physical activity and other lifestyle habits.

For dysthymia, the young and midlife women answered the Zung Self-Rating Depression Scale (SDS), whose cut-off value to considerate as normal is an SDS score below 40 [24, 25]. The older women completed the 30-item Geriatric Depression Scale (GDS) in which a woman with a score $\leq 10$ had not depression [26]. Major depression was diagnosed according to Diagnostic and Statistical Manual of Mental Disorders (DSM-5). Because both scales are different, the results were transformed into a normalized score, obtaining a percentage index multiplying the points obtained from each woman by 100 and dividing by 80 for SDS or 30 for GDS.
To evaluate sleep disturbances, we used the Athens Insomnia Scale (AIS), a validated psychometric instrument based on the ICD-10 criteria, designed to determine sleep difficulty. It is a self-assessment, in which a cut-off value of $\geq 8$ was considered as insomnia $[27,28]$.

Also, the women completed a structured questionnaire assessing the habits: smoking, intake of alcoholic and/or caffeinated beverages, and physical activity pointed type of activity, frequency, and duration, as lifestyle prooxidant factors. A prooxidant factor present was considerate if smoking $\geq 2$ cigarettes/day, intake of $\geq 2$ glasses/day alcoholic beverages (beer or spirits), intake of $>2$ cups/day highly caffeinated beverages, and $<30 \mathrm{~min} /$ day of physical activity (sedentary lifestyle).

2.5. Follow-Up Phase. All the measurements were carried out every 6 months for up to two years, to keep the sample. One person of our research group was in contact by phone with the participants each month during the follow-up time, ensuring that no women dropped out of the study, and they did not take hormone therapy or antioxidant supplements.

2.6. Statistical Analysis. Quantitative data were presented as the mean and standard deviation and categorical data as frequency, percentage, and $95 \%$ confidence interval $\left(\mathrm{CI}_{95 \%}\right)$. We used one-way ANOVA with Tukey's posthoc test and Pearson's chi-squared test, respectively, to compare them. We corroborate normal distribution of continuous data with the Kolgomorov-Smirnoff test and homogeneity of variances with the Levene test. To evaluate the proportions before and after two years, McNemar chi-squared test was used, and to 
TABLE 2: Physical activity performed at the beginning and after two years by study groups.

\begin{tabular}{|c|c|c|c|}
\hline Physical activity & Young women & Midlife women & Older women \\
\hline \multicolumn{4}{|c|}{ Walking $(\geq 30 \mathrm{~min} / \mathrm{d})$} \\
\hline Baseline & $4 / 59(7 \%, 1-14 \%)$ & $19 / 76(25 \%, 15-35 \%)^{\mathrm{a}}$ & $19 / 42(45 \%, 30-60 \%)^{\mathrm{b}, \mathrm{c}}$ \\
\hline After two years & $1 / 47(2 \%, 0-6 \%)$ & $16 / 78(21 \%, 12-30 \%)$ & $20 / 52(39 \%, 26-52 \%)^{\mathrm{d}}$ \\
\hline \multicolumn{4}{|l|}{ Aerobics $(60 \mathrm{~min} / \mathrm{d})$} \\
\hline Baseline & $7 / 59(12 \%, 4-20 \%)$ & $12 / 76(16 \%, 8-24 \%)$ & $3 / 42(7 \%, 3-11 \%)$ \\
\hline After two years & $6 / 47(13 \%, 3-23 \%)$ & $8 / 78(10 \%, 3-17 \%)$ & $7 / 52(13 \%, 4-22 \%)$ \\
\hline \multicolumn{4}{|l|}{ Yoga $(60 \mathrm{~min} / \mathrm{d})$} \\
\hline Baseline & $4 / 59(7 \%, 0-14 \%)$ & $4 / 76(5 \%, 0-8 \%)$ & $6 / 42(14 \%, 4-24 \%)$ \\
\hline After two years & $3 / 47(6 \%, 0-13 \%)$ & $7 / 78(9 \%, 3-15 \%)$ & $5 / 52(9 \%, 1-17 \%)$ \\
\hline \multicolumn{4}{|c|}{ Running ( $\geq 30 \mathrm{~min} / \mathrm{d})$} \\
\hline Baseline & $13 / 59(22 \%, 11-33 \%)$ & $5 / 76(7 \%, 1-13 \%)^{\mathrm{e}}$ & 0 \\
\hline After two years & $12 / 47(26 \%, 10-20 \%)$ & $6 / 78(8 \%, 2-14 \%)^{\mathrm{f}}$ & 0 \\
\hline \multicolumn{4}{|c|}{ Swimming $(60 \mathrm{~min} / \mathrm{d})$} \\
\hline Baseline & $4 / 59(7 \%, 1-14 \%)$ & 0 & 0 \\
\hline After two years & $3 / 47(6 \%, 0-13 \%)$ & $1 / 78(1 \%, 0-2 \%)$ & 0 \\
\hline \multicolumn{4}{|c|}{ Without physical activity } \\
\hline Baseline & $27 / 59(46 \%, 33-59 \%)$ & $36 / 76(47 \%, 36-58 \%)$ & $14 / 42(33 \%, 19-47 \%)^{g, h}$ \\
\hline After two years & $22 / 47(47 \%, 33-61 \%)$ & $40 / 78(51 \%, 40-62 \%)$ & $20 / 52(39 \%, 27-53 \%)$ \\
\hline
\end{tabular}

TABle 3: Baseline and after two years oxidative stress markers by study groups. Data show mean and standard deviation.

\begin{tabular}{|c|c|c|c|c|c|c|}
\hline Oxidative stress marker & $\begin{array}{l}\text { Young women } \\
\quad(n=59)\end{array}$ & $\begin{array}{l}\text { Baseline } \\
\text { Midlife women } \\
\quad(n=76)\end{array}$ & $\begin{array}{l}\text { Older women } \\
\quad(n=42)\end{array}$ & $\begin{array}{l}\text { Young women } \\
\quad(n=47)\end{array}$ & $\begin{array}{l}\text { After two years } \\
\text { Midlife women } \\
\quad(n=78)\end{array}$ & $\begin{array}{l}\text { Older women } \\
\quad(n=52)\end{array}$ \\
\hline Malondialdehyde $(\mu \mathrm{mol} / \mathrm{L})$ & $0.323 \pm 0.07$ & $0.336 \pm 0.07$ & $0.329 \pm 0.12$ & $0.328 \pm 0.06$ & $0.338 \pm 0.06$ & $0.341 \pm 0.09$ \\
\hline Superoxide dismutase $(\mathrm{U} / \mathrm{g} \mathrm{Hb})^{* \dagger}$ & $1.22 \pm 0.1$ & $1.19 \pm 0.1$ & $1.12 \pm 0.1$ & $1.27 \pm 0.2$ & $1.21 \pm 0.1^{\mathrm{a}}$ & $1.19 \pm 0.1^{\mathrm{b}}$ \\
\hline Glutathione peroxidase (U/g Hb) & $55.5 \pm 15.9$ & $53.3 \pm 16.5$ & $41.9 \pm 17.5$ & $55.7 \pm 16.5$ & $53.6 \pm 14.4$ & $46.9 \pm 19.0^{\mathrm{c}, \mathrm{d}}$ \\
\hline Uric acid $(\mu \mathrm{mol} / \mathrm{L})$ & $264 \pm 68$ & $275 \pm 76$ & $309 \pm 82^{\mathrm{d}}$ & $255 \pm 68$ & $297 \pm 74$ & $313 \pm 91^{\mathrm{e}}$ \\
\hline Total antioxidant status $(\mu \mathrm{mol} / \mathrm{L})^{\ddagger \S}$ & $1018 \pm 226$ & $1088 \pm 251$ & $1071 \pm 226$ & $1015 \pm 236$ & $1105 \pm 232$ & $913 \pm 188^{\mathrm{f}}$ \\
\hline Antioxidant gap $(\mu \mathrm{mol} / \mathrm{L})^{\ddagger \S}$ & $287 \pm 232$ & $338 \pm 239$ & $312 \pm 237$ & $311 \pm 247$ & $347 \pm 221$ & $156 \pm 251^{\mathrm{f}}$ \\
\hline SOD/GPx ratio & $0.024 \pm 0.007$ & $0.025 \pm 0.008$ & $0.031 \pm 0.012$ & $0.024 \pm 0.007$ & $0.024 \pm 0.007^{\mathrm{d}}$ & $0.029 \pm 0.010^{\mathrm{g}}$ \\
\hline Stress score & $3.42 \pm 1.85$ & $3.55 \pm 1.82$ & $4.36 \pm 1.51$ & $3.69 \pm 1.65$ & $3.46 \pm 1.77$ & $4.76 \pm 1.62^{\mathrm{e}, \mathrm{g}}$ \\
\hline
\end{tabular}

Repeated measures analysis of variance. ${ }^{*}$ Change over the time, $p<0.0001 ;{ }^{\dagger}$ age group-time interaction, $p<0.05 ;{ }^{*}$ change over the time, $p<0.05 ;{ }^{\S}$ age grouptime interaction, $p<0.01$. Tukey's test as posthoc. ${ }^{a}$ Young vs. midlife, $p<0.05$; ' young vs. older, $p<0.001$; ' young vs. older, $p<0.0001$; ${ }^{\mathrm{d}}$ midlife vs. older, $p$ $<0.001$; 'young vs. older, $p<0.01$; ${ }^{\mathrm{f}}$ midlife vs. older, $p<0.05$; ${ }^{\mathrm{g}}$ midlife vs. older, $p<0.0001$.

compare the means, paired t-test was calculated. A repeated measures multivariate analysis of variance was conducted to investigate the change of oxidative stress parameters over time. The between-subject factor was each age group, the within-subject factor was time (baseline versus 2 years), and Tukey's test was calculated as posthoc. Also, two factors repeated measures analysis of variance was carried out, adding to the model the dichotomous sedentary lifestyle as an another between-subject factor.

The multivariate technique of Principal Component Analysis (PCA) was used to systematically reduce the number of dimensions needed to describe OS. The prooxidant factors age, BMI, AIS score, dysthymia normalized score, number of cigarettes, cup of caffeine beverages, glasses of alcohol, and duration of physical activity were used to perform the PCA. Barlett's test of sphericity and the measure of sampling adequacy score of Kaiser-Meyer-Olkin (KMO) were obtained, to assess the homogeneity of variances among the different eigenvalues correlated to the principal component, and in which degree each variable may be predicted by all the other variables, respectively. A KMO value higher than 0.5 indicates that the solution obtained with PCA can be accepted. Because the data are not dimensionally homogeneous, the PCA was calculated with the method 
based on the correlation matrix, in which only the PCs eigenvalue greater than 1 was considerate, to assure that the set of PCs are more important than the original variables. In addition, an orthogonal rotation was performed by the varimax method to maximize the variance of the factors. When the correlation result of a factor was higher than 0.5 , it was considered highly correlated with a PC. A threedimensional plot was constructed in which the variables are positively correlated when they form an angle of 0 degrees from the origin and negatively when the angle is 180 degrees. An angle of 90 degrees indicates that the variables are uncorrelated $[29,30]$.

To assess the risk of each factor selected by the PCA, the Poisson regression test was used for multivariate analysis being as primary outcome the OS after 2 years, considering the cut-off value of $S S \geq 4$. The models were adjusted by the dichotomous variables smoking, intake of alcoholic beverages, intake of caffeinated beverages, overweight/obesity, dysthymia, insomnia, and sedentary lifestyle, as factors and age at baseline as quantitative covariable. The prevalence ratio (PR) and $\mathrm{CI}_{95 \%}$ were obtained. A risk factor was considerate if $\mathrm{PR}>1$ and the $95 \% \mathrm{CI}$ did not include the 1.0 values. A two-tailed $p$ value $<0.05$ was considerate as significant. The standard statistical software package SPSS V. 20.0 (IBM SPSS Statistics Armonk, NY, USA) was used for the processing of the data.

\section{Results}

3.1. Sample Characteristics. We observed that all the women completed the follow-up, and after 2 years, 12 (20\%) young women passed to the midlife age group, and $10(13 \%)$ midlife women passed to the older women group (Figure 1).

At basal time, young and midlife groups were similar in all the parameters, but the older women had higher glucose levels, and the proportion of diabetes mellitus and arterial hypertension is more frequent than the other groups. Regardless of the lifestyle factors, women who smoke or drink alcohol are few, with a prevalence ranging between 9 and $15 \%$ (Table 1).

The physical activity was different between the age group, the older and the midlife women frequently walk $\geq 30$ $\mathrm{min} / \mathrm{d}$, and the young women running $\geq 30 \mathrm{~min} / \mathrm{d}$. After two years, $95(54 \%)$ women still with physical activity, and 6 (12\%) old women became sedentary, but the change was not significant (Table 2).

3.2. Oxidative Stress by the Age Group. About oxidative stress markers, antioxidant enzymes were significantly lower in older women, and the SOD/GPx ratio and uric acid levels were higher, compared with young and midlife women. The malondialdehyde level did not change after two years. SOD activity, total antioxidant status, and antioxidant gap change over the time and shown an age group time interaction. The stress score is higher in older women than the other groups, and it increases after the two-year period in these women (Table 3).

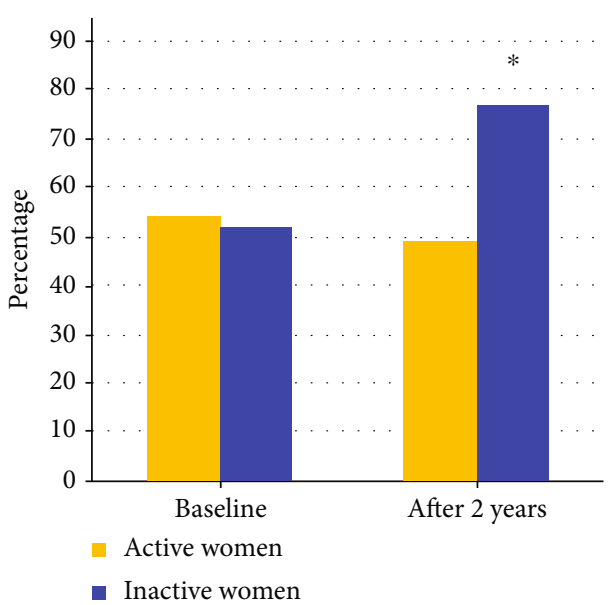

Figure 2: Percentage of women with oxidative stress at baseline and after two-year follow-up, separated by the active lifestyle. McNemar chi-squared test, ${ }^{*} p<0.001$.

3.3. Oxidative Stress and Sedentary Lifestyle. Concerning OS and sedentary lifestyle association, at baseline, 40 (52\%) of inactive women had high OS and after the two-year period, the proportion increased to 59 (77\%), $p<0.001$ (Figure 2). Likewise, stress score is higher in sedentary lifestyle women after the two-year follow-up; although, the difference was statistically significant only in older women; the OS score did not change in all groups of nonsedentary women (Figure 3).

To assess which prooxidant factors are most associated with OS, a PCA was performed. Preliminary analysis to verify the adequacy of the data for a factorial analysis showed a significant value of Barlett's test of sphericity $(p<0.0001)$ that allows formally contrasting the existence of correlation between the variables, and $\mathrm{KMO}=0.514$, which indicate that the PCA can be accepted. Four PC were selected, corresponding to $67.7 \%$ of the total variance, in which 7 out of 8 prooxidant factors distributed in the four PC and were important for the analysis. In Table 4, the main factors for OS are presented in order of importance, in which the correlation value approaching "- 1 or 1 " indicates that the variable significantly affects the component. BMI had low correlation; thus, it was discarded. Principal component 1 (PC1) included the dysthymia normalized score and age as higher correlation factors and accounted for $23 \%$ of the overall variance. The other three components accounted between 13\% and $17 \%$ of overall variance and included the other five prooxidant factors. The dysthymia normalized score and glasses of alcohol have a negative correlation.

In addition, the graphical representation of the component weights after rotation is shown in Figure 4. This plot shows the first three PCs which explains $\sim 55 \%$ of the total variance. As is difficult to see in a three-dimensional plot, the PC in which each variable is found, and a colored line was placed to indicate the PC to which it belongs. It is observed that the prooxidant factors: age (PC1), AIS scores (PC3), glasses of alcohol (PC4), and minutes of physical activity (PC4) form a cluster with small angles between each, and the dysthymia index (PC1) is on the opposite side. A 


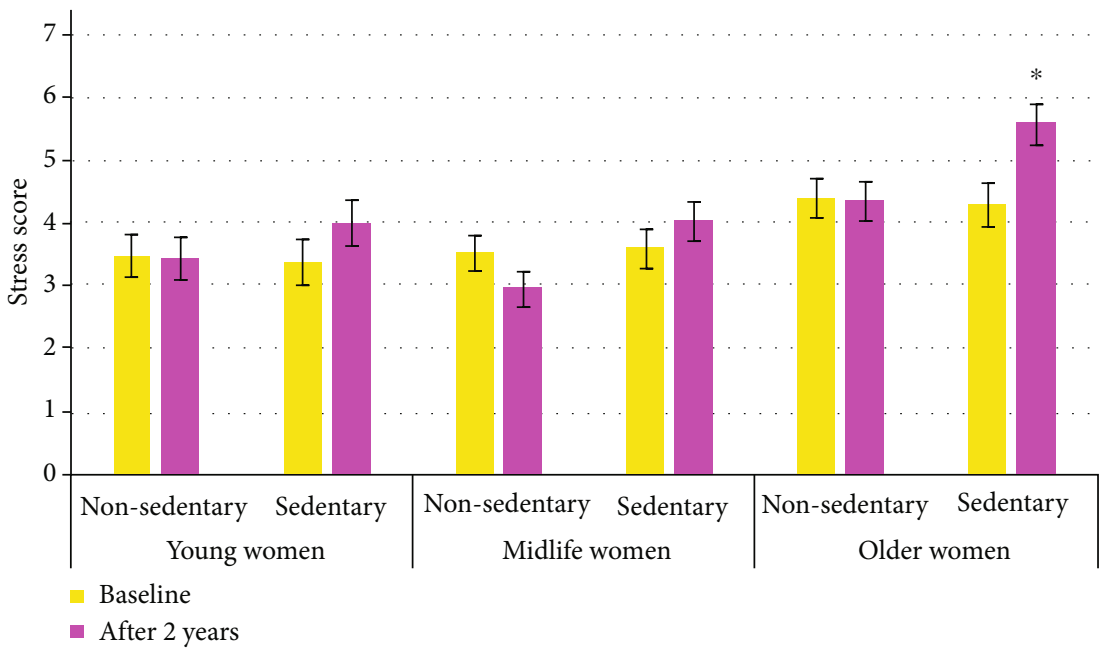

Figure 3: Stress scores by age groups at baseline and after two-year follow-up, separated by the sedentary lifestyle. Data shows mean and standard error. Two-way repeated measures ANOVA with paired $t$-test as posthoc, ${ }^{*} p<0.05$.

TABLE 4: Main factors to oxidative stress obtained by component principal analysis with orthogonal rotation.

\begin{tabular}{lccc}
\hline Principal component & Prooxidant factor & Correlation $r$ & Percentage of variance \% \\
\hline \multirow{2}{*}{1} & Age & 0.854 & 23.2 \\
& Dysthymia normalized score & -0.842 & 0.791 \\
3 & Cups of highly caffeinate beverage & 0.756 & 17.0 \\
3 & Number of cigarettes & 0.881 & 14.3 \\
4 & Athens insomnia scale score & 0.812 & 13.1 \\
\hline
\end{tabular}

second group is formed by number of cigarettes and cups of caffeinated beverages, and they are located in the PC2.

Because the PCA only indicates a cluster of prooxidant factors correlated with OS, but it is not easy to determinate the weight of each one, a Poisson regression was performed. In the risk analysis, the predictive factors for OS were the sedentary lifestyle (adjusted $\mathrm{PR}=2.37, \mathrm{CI}_{95 \%}$ : $1.30-4.30$, $p<0.01)$ and age, in which by each year of age that increases, the risk is $1.06\left(\mathrm{CI}_{95 \%}: 1.02-2.11, p<0.01\right)$, and other prooxidant factors did not risk for the OS (Table 5).

\section{Discussion}

Aging is a complex process that involves the time and the biological and environmental factors with the outcome of an imbalance between damage and repair of biomolecules, in which damage wins because the repair pathways gradually decline over the time, having the OS an important role as it participates in these reactions $[31,32]$. Being this a complex process, there is no direct relationship between OS and aging; so, there are external factors (prooxidants), such as lifestyle factors, that accelerate it due to a possible decrease in antioxidants and alteration of the immune system [33]. The role of sedentary lifestyle in aging OS relationship has been studied mainly in basic research $[16,34,35]$; therefore, it was our interest to assess the effect of this risk factor in the causal chain OS aging.
Most of the evidence shows that the physical activity changes across the life tending to a decrease during the aging process; however, also, an increment in leisure time physical activity is reported after the retirement transition, because the people have more time to do exercise [36], causing a controversial information. In women, it is recognized that after menopause, the physical functioning status decreases over the time, probably associated with physiological and symptomatic changes of this stage [11], causing a more sedentary lifestyle. Within this controversial information, the older women included in this study had more physical activity than the younger and middle-aged women; although, the exercise they perform is less impact since it is walking, compared with running or swimming. This is possible because, as we pointed out before, older women are concerned about their health and take on fewer social roles, different from younger and midlife people who are in a productive stage with high participation in the community [37]; thus, they have less time to dedicate for their health. Likewise, the benefit of the practice of moderate exercise is known to all. In this sense, exercise causes a hormetic response due an adaptive process to exposure to low quantities of stressors such as moderate physical activity, producing several beneficial effects like an improvement on cardiovascular function, and upregulates the immune system, delayed aging, and modulation of redox homeostasis with an increment of antioxidant response, among others [38, 39]; thus, the sedentary 


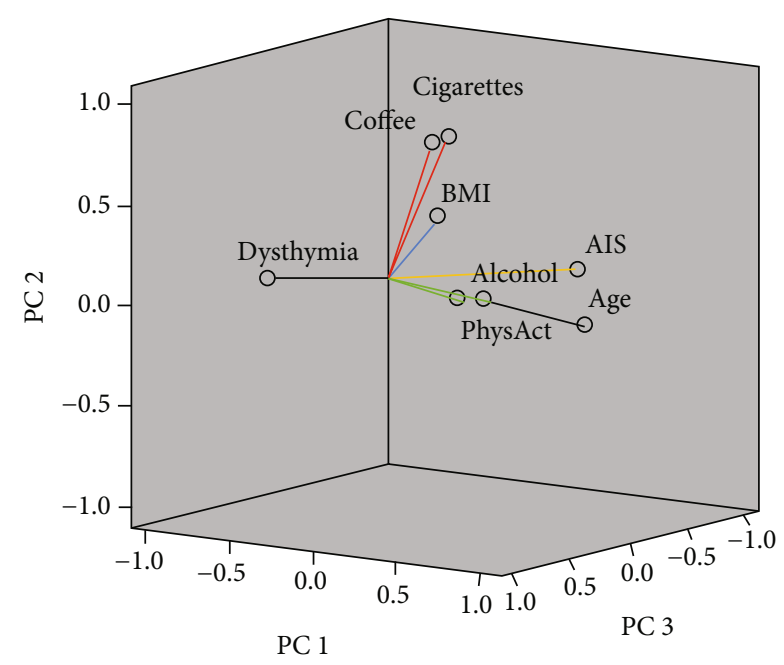

Figure 4: Three-dimensional plot of principal component analysis in rotate space notices how the variables age, AIS scores, glasses of alcohol, and minutes of physical activity form a cluster with small angles between each, and the dysthymia index is in the opposite side. A second group is formed by number of cigarettes and cups of caffeinated beverages. The variables with black lines are in $\mathrm{PC} 1$, with an orange line that is in $\mathrm{PC} 3$, with green lines that are in PC4, and with red lines that are in PC2. BMI had low correlation (blue line). AIS: Athens Insomnia Scale; alcohol: glasses of alcohol; BMI: body mass index; cigarettes: number of cigarettes; coffee: cups of caffeinated beverages; dysthymia: dysthymia index; PC: principal component; PhysAct: minutes of physical activity.

lifestyle has the opposite effect. Also, a review that analyzes the effect of prolonged sitting time on cardiometabolic risk recommended changing this behavior to a more active one to counteract the detrimental effect of sedentary lifestyle, according to the subjects' characteristics [40].

As we pointed above, the relationship between aging and OS had been described in several studies using different OS markers. In this study, we calculated an integrated stress score that involves both oxidized molecules and antioxidants, which corroborate that the OS is higher in older women, and after two years of follow-up, the OS only increases in this age group, corroborating the relationship, as previously it was reported in cross-sectional studies using individual markers [3,41]; although, a research has recently noted that only some OS markers change in adults over 55 years [42]. In this sense, in our study, lowest antioxidant components were observed in older women, but this is not necessarily indicative of the OS; therefore, it seems to point the importance to integrate the markers to define when a subject has an OS [43].

Several lifestyle factors are considered prooxidants because increase the OS. The PCA was performed to reduce the number of variables, because linear combination of the original variables is readily interpretable, therefore, each PC has a linear combination of the original variables [29]. This analysis revealed four PC containing 7 prooxidant factors. The PC1 explains the $23 \%$ of the variance, in which the age emerges as the main prooxidant factor positively associ- ated with the OS and negatively related to dysthymia. Concerning this, the OS increases with the age [3], and the dysthymia is more frequent in middle-aged women because they may be going through the menopausal transition, causing OS [9], that is why they are opposites in the same component. Secondly, AIS scores (PC3), glasses of alcohol (PC4), and minutes of physical activity (PC4) are close, as the threedimensional plot shows, indicating that these factors are interrelated, and they are not easy to separate to assess their effect. In addition, cups of caffeinated beverages and the number of cigarettes are in a second cluster group, and they are also variables to consider. The role of all those factors as prooxidants had been shown individually; although, the role of some remains unclear, and the association in a multivariate analysis is not common [44-47].

In PCA, the minutes of physical activity were introduced to the model, considering that our interest was to measure sedentary as a lifestyle with serious consequences during the aging. As well as the other factors, they are in a quantitative scale; thus, we transform all the variables in dichotomous scale to specify the risk to OS trying to separate the effect. In the risk analysis, only sedentary lifestyle and the age were risk factors for OS, and the other lifestyle prooxidant behaviors were not related. These results are consistent with the report of a study of factors associated with healthy aging of the Mexican population; the authors point out that smoking, alcohol intake, depression, and overweight/obesity are not related to the dependent variable [48]. Although a different association was sought, it seems that these variables are not related to events in which the age is included in the models. It is important to notice that age is the main risk factor for OS, independently if the analysis is with quantitative or categorical data, topic extensively discussed in the literature, but the aim of our study is to try to clarify the effect of the other pro-oxidant factors.

Likewise, our results have shown that the sedentary lifestyle increases the OS after a two-year period, mainly in older women, and it is a risk factor for OS. Thus, the women with sedentary lifestyle had twofold risk for OS, plus 6\% by each year of age; therefore, sedentary lifestyle seems to accelerate the OS during the aging, advancing the probability of tissue oxidative damage and incrementing the probability to develop the ageing-related diseases due to the OS, as previously has been indicated [49]. As for, it has been described that sedentary behavior is associated with mitochondrial oxidative damage in muscle [34] and enhances vascular reactive oxygen species production, which contributes to endothelial dysfunction and atherosclerosis [35]; hence, a sedentary lifestyle is associated with cardiovascular risk and loss of skeletal muscle mass and ageing-related events. Under such circumstances, it is recognized that a sedentary lifestyle is associated with depression, all-cause mortality, and cause of frailty in old people [50-52], events related to OS.

Furthermore, some studies showed that OS increases in elderly, although not significant, after 6 months of followup without intervention [53]. Therefore, the fact that with each year of age, the risk of OS increases besides sedentary lifestyle, and it is an additional contribution to studies of the 
TABle 5: Predictors of the outcome oxidative stress in 40 to 69 years women after a two-year period.

\begin{tabular}{lccc}
\hline Predictor & Prevalence ratio & $\mathrm{CI}_{95 \%}$ & \multicolumn{1}{c}{ value $^{*}$} \\
\hline Sedentary lifestyle (yes) & 2.37 & $1.30-4.30$ & 0.005 \\
Age (years) & 1.06 & $1.02-1.11$ & 0.003 \\
Smoking (yes) & 1.46 & $0.56-3.81$ & 0.438 \\
Alcohol beverages intake ( $\geq 2$ glasses/d) & 1.20 & $0.38-3.76$ & 0.751 \\
Dysthymia (yes) & 1.13 & $0.58-2.20$ & 0.725 \\
Highly caffeine beverages intake ( $\geq 2$ cups/d) & 0.94 & $0.52-1.71$ & 0.846 \\
Insomnia (yes) & 0.79 & $0.43-1.46$ \\
\hline
\end{tabular}

${ }^{*}$ Poisson regression, model $p$ value $<0.01 ; \mathrm{CI}_{95 \%}: 95 \%$ confidence interval.

harmful effect of this behavior and supports the research that has shown the benefit of low-impact exercise on OS and other health parameters $[19,54]$. In this context, it is important to highlight that in Mexico more than 65\% of the population aged 50 and over has a sedentary lifestyle [54].

The limitations of the study include the small sample size and the study follow-up time. Although it is important to highlight as strengths of the research the longitudinal design and the low drop of the participants of the cohort, in addition to the proposal to analyze the role of sedentary lifestyle instead of exercise. However, more longitudinal studies are needed to verify our findings.

\section{Conclusions}

It is important to note that in the two-year follow-up cohort of Mexican women in the aging process, the older subgroup (60 to 69 years) had significantly higher systolic blood pressure compared to the groups of young women (40 to 49 years) and middle-aged (50 to 59 years) $(p<0.001)$, in addition to a higher prevalence of diabetes mellitus in the older subgroup. Also, this group (60 to 69 years) was the one that reported a significantly higher percentage regard to continuing with physical activity $(p<0.001)$. Likewise, a significantly higher oxidative stress index was found in the subgroup of older women compared to the younger and middle-aged subgroups $(p<0.05)$. On the other hand, in the global analysis of the population, a positive correlation was observed between age, consumption of coffee cups, number of cigarettes, and score on the insomnia scale with the oxidative stress index, in contrast to a negative association with the moderate consumption of alcoholic beverages and the score on the scale to assess dysthymia. Finally, the most relevant finding of our study was that regardless of age and other prooxidant factors, sedentary lifestyle constitutes a statistically significant risk factor for oxidative stress Mexican women in the aging process.

These findings support the proposal to implement programs that strengthen healthy lifestyles during early aging.

\section{Data Availability}

The data used to support the findings of this study are available from the corresponding author upon request

\section{Disclosure}

The funders had no role in the design of the study; in collection, analyses, or interpretation of data; in the writing of the manuscript; or in the decision to publish the results.

\section{Conflicts of Interest}

The authors declare no conflict of interest.

\section{Acknowledgments}

The project was funded by the Dirección General de Asuntos del Personal Académico, Universidad Nacional Autónoma de México, grant number DGAPA-PAPIIT IN307220.

\section{References}

[1] D. Harman, "Aging: a theory based on free radical and radiation chemistry," Journal of Gerontology, vol. 11, no. 3, pp. 298-300, 1956.

[2] H. Sies, "Oxidative stress; concept and some practical aspects," Antioxidants, vol. 9, no. 9, p. 852, 2020.

[3] V. M. Mendoza-Núñez, M. Ruiz-Ramos, M. A. SánchezRodríguez, R. Retana-Ugalde, and J. L. Muñoz-Sánchez, "Aging-related oxidative stress in healthy humans," Tohoku Journal of Experimental Medicine, vol. 213, no. 3, pp. 261268, 2007.

[4] I. Pinchuk, D. Weber, B. Kochlik et al., "Gender- and agedependencies of oxidative stress, as detected based on the steady state concentrations of different biomarkers in the MARK-AGE study," Redox Biology, vol. 24, article 101204, 2019.

[5] S. Dato, P. Crocco, P. D'Aquila et al., "Exploring the role of genetic variability and lifestyle in oxidative stress response for healthy aging and longevity," International Journal of Molecular Sciences, vol. 14, no. 8, pp. 16443-16472, 2013.

[6] F. J. Broekmans, M. R. Soules, and B. C. Fauser, "Ovarian aging: mechanisms and clinical consequences," Endocrine Reviews, vol. 30, no. 5, pp. 465-493, 2009.

[7] N. E. Avis, S. Brockwell, and A. Colvin, "A universal menopausal syndrome?," The American Journal of Medicine, vol. 118, no. 12, pp. 37-46, 2005.

[8] S. B. Doshi and A. Agarwal, "The role of oxidative stress in menopause," Journal of Mid-life Health, vol. 4, no. 3, pp. 140-146, 2013.

[9] M. A. Sánchez-Rodríguez, L. Castrejón-Delgado, M. ZacaríasFlores, A. Arronte-Rosales, and V. M. Mendoza-Núñez, 
"Quality of life among post-menopausal women due to oxidative stress boosted by dysthymia and anxiety," BMC Womens Health, vol. 17, no. 1, p. 1, 2017.

[10] M. A. Sánchez-Rodríguez, M. Zacarías-Flores, A. ArronteRosales, and V. M. Mendoza-Núñez, "Association between hot flashes severity and oxidative stress among Mexican postmenopausal women: a cross-sectional study," PLoS One, vol. 14, no. 9, article e0214264, 2019.

[11] L. A. Tseng, S. R. el Khoudary, E. A. Young et al., "The association of menopause status with physical function: the study of Women's health across the nation," Menopause, vol. 19, no. 11, pp. 1186-1192, 2012.

[12] G. Lessiani, F. Santilli, A. Boccatonda et al., "Arterial stiffness and sedentary lifestyle: role of oxidative stress," Vascular Pharmacoloty, vol. 79, pp. 1-5, 2016.

[13] Y. Kim, K. Wijndaele, S. J. Sharp et al., "Specific physical activities, sedentary behaviours and sleep as long-term predictors of accelerometer-measured physical activity in 91, 648 adults: a prospective cohort study," The International Journal of Behavioral Nutrition and Physical Activity, vol. 16, no. 1, p. 41, 2019.

[14] A. Loyen, J. Y. Chau, J. G. M. Jelsma, F. van Nassau, and H. P. van der Ploeg, "Prevalence and correlates of domain-specific sedentary time of adults in the Netherlands: findings from the 2006 Dutch time use survey," BMC Public Health, vol. 19, supplement 2, p. 538, 2019.

[15] A. Safdar, M. J. Hamadeh, J. J. Kaczor, S. Raha, J. DeBeer, and M. A. Tarnopolsky, "Aberrant mitochondrial homeostasis in the skeletal muscle of sedentary older adults," PLOS ONE, vol. 5, no. 5, article e10778, 2010.

[16] D. M. Bailey, J. McEneny, O. Mathieu-Costello et al., "Sedentary aging increases resting and exercise-induced intramuscular free radical formation," Journal of Applied Physiology, vol. 109, no. 2, pp. 449-456, 2010.

[17] M. A. Sánchez-Rodríguez, V. M. Mendoza-Núñez, A. GarcíaSánchez, B. González-González, E. Rodríguez-Torres, and A. González-Obregón, "Valores de referencia de poblaciones senecta y adulta de la ciudad de México. Parámetros bioquímicos y hematológicos," Acta Bioquímica Clínica Latinoamericana, vol. 32, no. 3, pp. 397-405, 1998.

[18] M. D. Jensen, D. H. Ryan, C. M. Apovian et al., “2013 AHA/ACC/TOS Guideline for the Management of Overweight and Obesity in Adults: A Report of the American College of Cardiology/American Heart Association Task Force on Practice Guidelines and The Obesity Society," Journal of the American College of Cardiology, vol. 63, no. 25 Part B, pp. 2985-3023, 2014.

[19] V. M. Mendoza-Núñez, T. L. Arista-Ugalde, J. Rosado-Pérez, M. Ruiz-Ramos, and E. Santiago-Osorio, "Hypoglycemic and antioxidant effect of tai chi exercise training in older adults with metabolic syndrome," Clinical Interventions in Aging, vol. 13, pp. 523-531, 2018.

[20] D. Machin, M. J. Campbell, S. B. Tan, and S. H. Tan, Sample Size Tables for Clinical Studies, United Kingdom, John Wiley \& Sons, 2009.

[21] A. M. Jentzsch, H. Bachmann, P. Fürst, and H. K. Biesalski, "Improved analysis of malondialdehyde in human body fluids," Free Radical Biology \& Medicine, vol. 20, no. 2, pp. 251-256, 1996.

[22] N. J. Miller, "Nonvitamin plasma antioxidants," in Free Radical and Antioxidant Protocols, D. Armstrong, Ed., vol. 108, pp. 285-298, Humana Press, New Jersey, USA, 1998.
[23] M. A. Sánchez-Rodríguez, M. Ruiz-Ramos, and V. M. Mendoza-Núñez, "Proposal of a construct to measure severity of oxidative stress," Free Radical Biology \& Medicine, vol. 41, supplement 1, p. S29, 2006.

[24] W. W. Zung, C. B. Richards, and M. J. Short, "Self-rating depression scale in an outpatient Clinic," Archives on General Psychiatry, vol. 13, no. 6, pp. 508-515, 1965.

[25] B. J. Carroll, J. Fielding, and T. G. Blashki, "Depression rating scales: a critical review," Archives on General Psychiatry, vol. 28, no. 3, pp. 361-366, 1973.

[26] J. A. Yesavage, T. L. Brink, T. L. Rose et al., "Development and validation of a geriatric depression screening scale: A preliminary report," Journal of Psychiatric Research, vol. 17, no. 1, pp. 37-49, 1982.

[27] C. R. Soldatos, D. G. Dikeos, and T. J. Paparrigopoulos, "Athens insomnia scale: validation of an instrument based on ICD10 criteria," Journal of Psychosomatic Research, vol. 48, no. 6, pp. 555-560, 2000.

[28] C. R. Soldatos, D. G. Dikeos, and T. J. Paparrigopoulos, "The diagnostic validity of the Athens insomnia scale," Journal of Psychosomatic Research, vol. 55, no. 3, pp. 263-267, 2003.

[29] I. T. Jolliffe and J. Cadima, "Principal component analysis: a review and recent developments," Philosophical Transactions Royal Society A, vol. 374, no. 2065, 2016.

[30] T. D. Martins, J. M. Annichino-Bizzacchi, A. V. C. Romano, and R. M. Filho, "Principal component analysis on recurrent venous thromboembolism," Clinical and Applied Thrombosis/Hemostasis, vol. 25, 2019.

[31] S. Dodig, I. Čepelak, and I. Pavić, "Hallmarks of senescence and aging," Biochemia Medica (Zagreb), vol. 29, no. 3, article 030501, 2019.

[32] M. C. Haigis and B. A. Yankner, "The aging stress response," Molecular Cell, vol. 40, no. 2, pp. 333-344, 2010.

[33] I. Peluso, M. Palmery, N. S. Yarla, G. Perry, and M. A. Kamal, "From oxidative stress to ageing via lifestyle, nutraceuticals, polypharmacy, and neuropsychological factors," Oxidative Medicine and Cellular Longevity, vol. 2018, Article ID 6352689, 2 pages, 2018.

[34] P. A. Figueiredo, S. K. Powers, R. M. Ferreira, F. Amado, H. J. Appell, and J. A. Duarte, "Impact of lifelong sedentary behavior on mitochondrial function of mice skeletal muscle," The Journals of Gerontology. Series A, Biological Sciences and Medical Sciences, vol. 64A, no. 9, pp. 927-939, 2009.

[35] U. Laufs, S. Wassmann, T. Czech et al., "Physical inactivity increases oxidative stress, endothelial dysfunction, and atherosclerosis," Arteriosclerosis, Thrombosis Vascular Biology, vol. 25, no. 4, pp. 809-814, 2005.

[36] I. Barnett, E. M. F. van Sluijs, and D. Ogilvie, "Physical Activity and Transitioning to Retirement: A Systematic Review," American Journal of Preventive Medicine, vol. 43, no. 3, pp. 329-336, 2012.

[37] H. L. Giasson, T. L. Queen, M. Larkina, and J. Smith, “Age group differences in perceived age discrimination: associations with self-perceptions of aging," Gerontologist, vol. 57, supplement 2, pp. S160-S168, 2017.

[38] Z. Radak, H. Y. Chung, and S. Goto, "Exercise and hormesis: oxidative stress-related adaptation for successful aging," Biogerontology, vol. 6, no. 1, pp. 71-75, 2005.

[39] Z. Radak, H. Y. Chung, E. Koltai, A. W. Taylor, and S. Goto, "Exercise, oxidative stress and hormesis," Ageing Research Reviews, vol. 7, no. 1, pp. 34-42, 2008. 
[40] M. Kozakiewicz, M. Kornatowski, O. Krzywińska, and K. Kędziora-Kornatowska, "Changes in the blood antioxidant defense of advanced age people," Clinical Interventions in Aging, vol. 14, pp. 763-771, 2019.

[41] D. Weber, W. Stuetz, O. Toussaint et al., “Associations between specific redox biomarkers and age in a large European cohort: the MARK-AGE project," Oxidative Medicine and Cellular Longevity, vol. 2017, Article ID 1401452, 12 pages, 2017.

[42] F. B. Benatti and M. Ried-Larsen, "The effects of breaking up prolonged sitting time: a review of experimental studies," Medicine and Science in Sports and Exercise, vol. 47, no. 10, pp. 2053-2061, 2015.

[43] M. A. Sánchez-Rodríguez and V. M. Mendoza-Núñez, “Oxidative stress indexes for diagnosis of health or disease in humans," Oxidative Medicine and Cellular Longevity, vol. 2019, Article ID 4128152, 32 pages, 2019.

[44] B. Liang, Y.-H. Li, and H. Kong, "Serum paraoxonase, arylesterase activities and oxidative status in patients with insomnia," European Review for Medical and Pharmacological Sciences, vol. 17, no. 18, pp. 2517-2522, 2013.

[45] D. Martini, C. del Bo', M. Tassotti et al., "Coffee consumption and oxidative stress: a review of human intervention studies," Molecules, vol. 21, no. 8, p. 979, 2016.

[46] H. K. Tan, E. Yates, K. Lilly, and A. D. Dhanda, "Oxidative stress in alcohol-related liver disease," World Journal of Hepatology, vol. 12, no. 7, pp. 332-349, 2020.

[47] S. Dikalov, H. Itani, B. Richmond et al., "Tobacco smoking induces cardiovascular mitochondrial oxidative stress, promotes endothelial dysfunction, and enhances hypertension," American Journal of Physiology. Heart and Circulatory Physiology, vol. 316, no. 3, pp. H639-H646, 2019.

[48] C. Arroyo-Quiroz, R. Brunauer, and S. Alavez, "Factors associated with healthy aging in septuagenarian and nonagenarian Mexican adults," Maturitas, vol. 131, pp. 21-27, 2020.

[49] B. L. Tan, M. E. Norhaizan, W. P. Liew, and H. Sulaiman Rahman, "Antioxidant and oxidative stress: a mutual interplay in age-related diseases," Frontiers in Pharmacology, vol. 9, p. 1162, 2018.

[50] L. K. Wilson-Escalante, M. A. Sánchez-Rodríguez, and V. M. Mendoza-Núñez, "Sedentarismo como factor de riesgo de trastornos depresivos en adultos mayores. Un estudio exploratorio," Revista de la Facultad de Medicina UNAM, vol. 52, no. 6, pp. 244-247, 2009.

[51] P. von Rosen, I. M. Dohrn, and M. Hagströmer, “Association between physical activity and all-cause mortality: a 15-year follow-up using a compositional data analysis," Scandinavian Journal of Medicine \& Science in Sports, vol. 30, no. 1, pp. 100-107, 2020.

[52] A. Mañas, B. del Pozo-Cruz, I. Rodríguez-Gómez et al., "Doseresponse association between physical activity and sedentary time categories on ageing biomarkers," BMC Geriatrics, vol. 19, no. 1, p. 270, 2019.

[53] J. Rosado-Pérez, R. Ortiz, E. Santiago-Osorio, and V. M. Mendoza-Núñez, "Effect of tai chi versus walking on oxidative stress in Mexican older adults," Oxidative Medicine and Cellular Longevity, vol. 2013, Article ID 298590, 8 pages, 2013.

[54] C. Daskalopoulou, A. Koukounari, Y. T. Wu et al., "Healthy ageing trajectories and lifestyle behaviour: the Mexican health and aging study," Scientific Reports, vol. 9, no. 1, p. 11041, 2019. 